\title{
Smac/DIABLO regulates the apoptosis of hypertrophic scar fibroblasts
}

\author{
BAO-HENG LIU*, LIANG CHEN**, SHI-RONG LI, ZHEN-XIANG WANG and WEN-GUANG CHENG \\ Department of Plastic and Reconstructive Surgery, Southwest Hospital, \\ The Third Military Medical University, Chongqing 400038, P.R. China
}

Received March 13, 2013; Accepted June 10, 2013

DOI: $10.3892 /$ ijmm.2013.1442

\begin{abstract}
In abnormal skin wound healing, hypertrophic scars (HS) are characterized by excessive fibroblast hypercellularity and an overproduction of collagen, leading to atypical extracellular matrix (ECM) remodeling. Although the exact mechanisms of HS remain unclear, decreased HS fibroblast (HSFB) apoptosis and increased proliferation are evident in the development of HS. In this study, the contribution of the second mitochondria-derived activator of caspases/direct inhibitor of apoptosis protein (IAP)-binding protein with a low isoelectric point (pI) (Smac/DIABLO), an apoptosis-promoting protein released from the mitochondria, was investigated in human normal skin and HSFB cultures. The expression of Smac/ DIABLO is usually decreased in many malignant tumors compared with normal tissues. Immunohistochemical analysis of skin tissues and the western blot analyses of fibroblasts revealed that the expression of Smac/DIABLO was lower in HS tissues compared with normal skin tissues. Of note, adenovirusmediated Smac/DIABLO overexpression in the cultured HSFBs significantly reduced cell proliferation, as detected by the cell counting kit- 8 , and increased caspase- 3 and -9 activity, as detected by spectrofluorimetry. In addition, it increased apoptosis, as detected by fluorescence-activated cell sorting (FACS). Furthermore, we found that the silencing of Smac with siRNA in the HSFBs induced a noticeable decrease in caspase- 3 and -9 activity, leading to a significant reduction in apoptosis. In addition, the mRNA expression of type I and III pro-collagen detected in the HSFBs was significantly increased following the silencing of Smac with siRNA and was inhibited following Smac/DIABLO overexpression, as shown by real-time RT-PCR. In conclusion, Smac/DIABLO decreases the proliferation and increases the apoptosis of HSFBs. To our knowledge, the data
\end{abstract}

Correspondence to: Professor Shi-Rong Li, Department of Plastic and Reconstructive Surgery, Southwest Hospital, The Third Military Medical University, 29 Gaotanyan Main Street, Shapingba, Chongqing 400038, P.R. China

E-mail: lishirong.xnzx@yahoo.com.cn

*Contributed equally

Key words: hypertrophic scar, apoptosis, Smac, DIABLO, fibroblast from our study suggest for the first time that Smac/DIABLO is a novel therapeutic target for HS.

\section{Introduction}

The formation of hypertrophic scars (HS) is a common abnormal response to wound healing which generally occurs following surgery, trauma and burns. Patients with HS often have severe impairment of their quality of life, including itching, pain, stiffness, loss of joint mobility or anatomical deformities that finally delay the resumption of normal life and create a huge financial burden on the healthcare system. Many treatments for HS, such as surgical excision, corticosteroid injections, splinting and pressure therapy, as well as novel methods, including interferon and 5-fluorouracil therapy, are not often completely successful and HS easily recur. There is still no consensus regarding the most effective treatment to completely and permanently improve scars with minimal side-effects (1). Currently, little is known about the incidence and risk factors for HS, although histologically, HS are characterized by excessive fibroblast hypercellularity, an overproduction of collagen and atypical extracellular matrix (ECM) remodeling in the scar tissue (2). Although the exact mechanisms of HS remain unclear, decreased fibroblast apoptosis in the wound bed and consequently, increased proliferation are observed during the development of HS (3).

Normal wound healing requires the orchestrated recruitment and proliferation of various cell types in wounds followed by their rapid disappearance. Thus, following re-epithelialization, contraction and when sufficient ECM is formed, the myofibroblast phenotype normally disappears mainly by the activation of programmed cell death (4). Simultaneously, inflammatory cells are also eliminated to allow the formation of granulation tissue. However, in HS, myofibroblasts and inflammatory cells tend to persist, which may represent an important element in the mechanisms of excessive ECM deposition and scar contractures. Previous studies have suggested that the decrease in myofibroblast sensitivity in response to apoptosis leads to an imbalance between ECM deposition and degradation, resulting in HS $(4,5)$. While apoptosis is believed to play an important role in these processes, its upstream regulators and effectors in the wound environment remain unclear. Certain studies have described a decrease in apoptosis together with higher resistance to apoptotic inducers in HS myofibroblasts (6), whereas others have found an increased rate of apoptosis $(7,8)$. 
The focal upregulation of p53 expression, which plays an important role in the inhibition of apoptosis, has been reported in situations of excessive scarring (9). There is evidence that Akt mediates this process through the phosphorylation of Bad (9), a negative regulator of $\mathrm{Bcl}-2$, which downregulates apoptosomal activation by Bax and Bak, leading to the inhibition of the intrinsic pathway of apoptosis. In this pathway, imbalance between pro(Bax and Bak) and anti-apoptotic (Bcl-2 and $\mathrm{Bcl}-\mathrm{xL}$ ) proteins on the mitochondrial membrane stimulates the release of several mitochondrial proteins, including cytochrome $c$, apoptosisinducing factor, endonuclease $\mathrm{G}$ and second mitochondria derived activator of caspase/direct inhibitor of apoptosis protein (IAP)-binding protein with a low isoelectric point (pI) (Smac/ DIABLO) $(10,11)$. In the cytosol, cytochrome $c$, apoptotic peptidase activating factor-1 (Apaf-1) and pro-caspase-9 form the apoptosome complex which activates primary downstream targets (i.e., pro-caspase-3). Finally, active caspases induce characteristic apoptotic changes through their ability to cleave certain key protein substrates in the cell. These caspases can be inhibited by IAPs through direct binding with the baculovirus inhibitory repeat (BIR) domain of IAPs. Of note, little is known about the wound healing effects of the Smac/DIABLO protein, which regulates caspase inhibition by IAPs. Previous studies have described the ability of the Smac/DIABLO protein to downregulate cell proliferation and promote cell apoptosis (12). The expression of Smac has been shown to be downregulated in renal cell carcinoma $(13,14)$, lung cancer $(15)$, testicular germ cell tumors (16) and hepatocellular carcinoma (17). Moreover, an inverse correlation has been found between the expression levels of Smac/DIABLO and cancer progression (18).

As several studies have confirmed that Smac/DIABLO promotes apoptosis, we hypothesized that Smac/DIABLO may be involved in the formation of HS. In this study, we examined Smac/DIABLO expression in HS and the effects of its overexpression in HS fibroblasts (HSFBs).

\section{Materials and methods}

Patient specimens. HS and normal skin tissues were obtained from 25 subjects (11 males and 14 females, aged 2-34 years) who underwent scar resection at 6-12 months following severe empyrosis. This study was approved by the Ethics Committee of Southwest Hospital of the Third Military Medical University (Chongqing, China). HS tissues were erythematous, raised, pruritic and confined to the site of injury. All samples were in the actively hyperplastic phase, as confirmed by pathological examination. Local infection, ulceration and treatment with glucocorticosteroids or radiotherapy were excluded. Informed consent was obtained from each participant.

Cell cultures. Fibroblasts were established as primary cell lines from HS and normal skin tissue. Using sterile techniques under a laminar flow hood, normal skin and HS tissues were washed in triplicate in Hank's solution, and the epidermis and subcutaneous fat layer were then removed. The dermal specimen was minced into pieces (sized $0.5-1.0 \mathrm{~mm}^{3}$ ) using a sterile scalpel blade on a Petri dish. All specimens were washed with phosphate-buffered saline (PBS) solution with a combination of $1 \%$ penicillin, amphotericin $\mathrm{B}$ and streptomycin sulfate. In order to allow the tissue pieces to adhere to the flask wall firmly, the specimens were cultured in serum-containing medium $\left(37^{\circ} \mathrm{C}\right.$, $5 \% \mathrm{CO}_{2}$ ) consisting of DMEM, $10 \%$ fetal bovine serum and $1 \%$ penicillin-streptomycin. The medium was changed every 2-3 days until the fibroblasts were grew to a monolayer and were spread over the flask bottom when observed under a light microscope. The tissues were then removed and the cells were subcultured. The culture medium was changed every 4 days, and successive subcultures were performed upon confluence. The growth status and morphological changes of the cells were observed under an inverted microscope. Early-passage cells (passages 3-6) were used in this study. Normal skin fibroblasts (NSFBs) were used as the controls for the HSFBs from the same donors, to confirm that HSFBs acted differently from normal fibroblasts.

Immunohistochemistry of Smac. HS specimens and normal skin specimens were fixed, embedded and cut into $\sim 5-\mu$ m-thick sections that were placed on Aptex-coated slides. They were then dewaxed in xylene and rehydrated after $10 \mathrm{~min}$ on heat. Blocking conditions were then performed for $30 \mathrm{~min}$. The primary antibody for Smac (1:500; Bioss Inc., Beijing, China) was then added followed by incubation for $1.5 \mathrm{~h}$. The secondary antibody was then added coupled with avidin (1:200; Bioss Inc.). We examined the tissues under an Olympus microscope and SPOT ${ }^{\mathrm{TM}}$ digital microscope camera (Diagnostic Instruments, Inc., Sterling Heights, MI, USA).

Western blot analysis of Smac. HSFBs and NSFBs were lysed immediately with cold lysis buffer containing $1 \%$ phenylmethylsulfonyl fluoride, $1 \%$ protease inhibitor cocktail and $1 \%$ sodium orthovanadate (Santa Cruz Biotechnology, Inc., Santa Cruz, CA, USA). The supernatants were harvested following centrifugation. Protein was collected using the BCA protein assay kit according to the manufacturer's instructions (Beyotime, Shanghai, China). Proteins were separated by $10 \%$ SDS-PAGE and transferred onto polyvinylidene difluoride membranes. After blocking with $5 \%$ non-fat milk, the membranes were incubated with an antibody against Smac (1:200; Santa Cruz Biotechnology, Inc.) overnight at $4^{\circ} \mathrm{C}$. The membranes were washed with TBS in triplicate and then incubated with secondary antibody for $1 \mathrm{~h}$ at room temperature. The protein-antibody complex was visualized by electrochemiluminescence (ECL) western blotting detection reagents. $\beta$-actin was used as the control. The gray scale densities of $\beta$-actin and Smac were assayed using ImageJ software.

Overexpression and silencing of Smac/DIABLO. The Smac/ DIABLO gene was purchased from ProteinTech Group, Inc. (Wuhan, China). We selected Sal1 and Xba1 as the cutting sites. The primers used for the PCR amplification of Smac/ DIABLO were: Sal1, 5'-ACGCGTCGACATGGCGGCTCT GAAGAGTTGG-3'; and Xba1, 5'-GCTCTAGATCAATCCT CACGCAGGTAGGC-3'. Adenovirus carrying the Smac/ DIABLO gene with an enhanced green fluorescent protein was used as the overexpression group (AD-Smac group), and adenovirus carrying green fluorescent protein only was used as the control (control AD-Smac group). Fibroblasts incubated with PBS only were also used as the blank controls (HSFB group). The HSFBs were incubated with adenovirus at various multiplicities of infection for $24 \mathrm{~h}$. Three days later, protein 

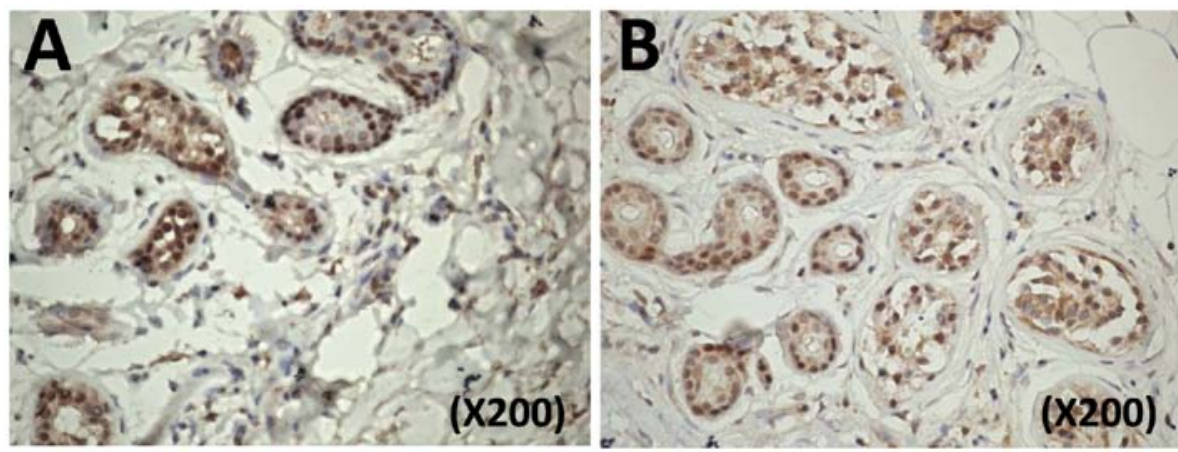

Figure 1. Immunohistochemical staining of Smac protein in skin tissue. Hypertrophic scar (HS) specimens and normal skin specimes from 25 subjects were fixed and stained with anti-Smac antibody. (A) A relatively large amount of Smac protein was detected in the NS tissue. (B) By contrast, Smac was minimally detected and localized in the nucleus and cytoplasm in the HS samples.

expression was detected by both fluorescence microscopy and flow cytometry.

Smac siRNA was purchased from Santa Cruz Biotechnology, Inc. The control siRNA had the same composition of nucleotides, but there was no homology between the control siRNA and Smac mRNA. The HSFBs were inoculated in a 24-well plate and incubated for a whole day, then transfected with Smac siRNA (siRNA group), or with control siRNA (control siRNA group). The blank control was HSFBs incubated with PBS (HSFB group).

Cell proliferation analysis. We used the cell counting kit- 8 (CCK-8) assay, which is widely used to quantify cell proliferation, to assess HSFB proliferation following transfection with AD-Smac. The detection sensitivity of CCK-8 is higher than other tetrazolium salts, such as 3-(4,5-dimethylthiazol-2-yl)-2, 5-diphenyltetrazolium bromide (MTT), 2,3-bis-(2-methoxy-4nitro-5-sulfophenyl)-2H-tetrazolium-5-carboxanilide (XTT), 3-(4,5-dimethylthiazol-2-yl)-5-(3-carboxymethoxyphenyl)-2(4-sulfophenyl)-2H-tetrazolium (MTS) or water-soluble tetrazolium salt (WST-1). We inoculated the HSFB suspension (100 $\mu \mathrm{l} /$ well) in a 96-well plate, then pre-incubated the plate in a humidified incubator $\left(37^{\circ} \mathrm{C}, 5 \% \mathrm{CO}_{2}\right)$ for $24 \mathrm{~h}$. We then added $10 \mu \mathrm{l}$ of the CCK-8 solution to each well followed by incubation for $1 \mathrm{~h}$. The absorbance was measured at $450 \mathrm{~nm}$ using a microplate reader (Flash Spectrum Biology Technology Co., Ltd.; Shanghai, China).

Detection of apoptosis and cell cycle analysis. For the detection of apoptosis, the HSFBs transfected with AD-Smac or the control were cultured in 6-well plates for $24 \mathrm{~h}$. Apoptosis was then detected using the terminal deoxynucleotidyl transferase dUTP nick end-labeling (TUNEL) assay kit, according to the instructions of the manufacturer (Promega, Madison, WI, USA) and the cell cycle of the HSFBs was analyzed using a FACSAria flow cytometer (BD Biosciences, San Jose, CA, USA). The data were analyzed using CellQuest 3.0 software (BD Biosciences).

mRNA levels of type I and III pro-collagen. The levels of type I and III pro-collagen were quantified by real-time RT-PCR. Primers used were as follows: type I pro-collagen forward, 5'-GTTCGTCCTTCTCAGGGTAG-3' and reverse, 5'-TTGTCGTAGCAGGGTTCTTT-3'; fragment length, 254 bp; type III pro-collagen forward, 5'-CGAGGTAACAGAGG TGAAAGA-3' and reverse, 5'-AACCCAGTATTCTCCACT CTT-3'; fragment length, $349 \mathrm{bp}$; human $\beta$-actin forward, 5'-TCCCTGGAGAAGAGCTACGA-3' and reverse, 5'-AGCA CTGTGTTGGCGTACAG-3'. The initial denaturation was achieved at $94^{\circ} \mathrm{C}$ for $2 \mathrm{~min}$, denaturation at $94^{\circ} \mathrm{C}$ for $30 \mathrm{sec}$, reassociation at $55^{\circ} \mathrm{C}$ for $30 \mathrm{sec}$, extension at $72^{\circ} \mathrm{C}$ for $30 \mathrm{sec}$ (28 cycles) and a final extension step at $72^{\circ} \mathrm{C}$ for $10 \mathrm{~min}$.

Caspase- 3 and -9 activity. For caspase- 3 or -9 activity assays in the HSFBs, each reaction with a final volume of $30 \mu \mathrm{l}$ was assembled on ice, including caspase-3 substrate (Ac-DEVD-AFC, $1 \mathrm{mM}$ ) or caspase-9 substrate (Ac-LEHD-AFC, $1 \mathrm{mM}$ ) and 10X caspase assay buffer. The generation of a fluorescence signal expressed in relative fluorescent units, due to the cleavage of substrates by caspase-3 or -9 , was measured using an automated spectrophotometer (Shanghai Metash Instruments Co., Ltd., Shanghai, China) at wavelengths of $360 / 465$ or $400 / 505 \mathrm{~nm}$ (excitation/emission).

Statistical analyses. Experiments were conducted in triplicate and data are expressed as the means \pm standard error. The Student's t-test was performed to evaluate group differences with SPSS 11.0 statistical software (SPSS Inc., Chicago, IL, USA). Values of $\mathrm{P}<0.05$ were considered to represent statistically significant differences.

\section{Results}

Smac expression is lower in HS and HSFBs than normal tissue and fibroblasts. Smac expression as detected by immunohistochemistry in the NSFBs was stronger than in the HS, where a weak expression was detected (Fig. 1). Following western blot analysis of the cell extracts, the Smac protein band at $25 \mathrm{kDa}$ was more prominent in the NSFBs than in the HSFBs (Fig. 2). Using ImageJ software, quantitative analyses of Smac expression in 25 patients indicated and confirmed that the relative Smac protein level corresponding to the ratio of Smac/ $\beta$-actin was significantly lower $(11.03 \%)$ in the HSFBs than in the NSFBs $(25.02 \%)(\mathrm{P}<0.01)$.

Smac overexpression induces apoptosis of HSFBs. As Smac expression is low in HSFBs, we explored the modifications induced by the overexpression and suppression of Smac 

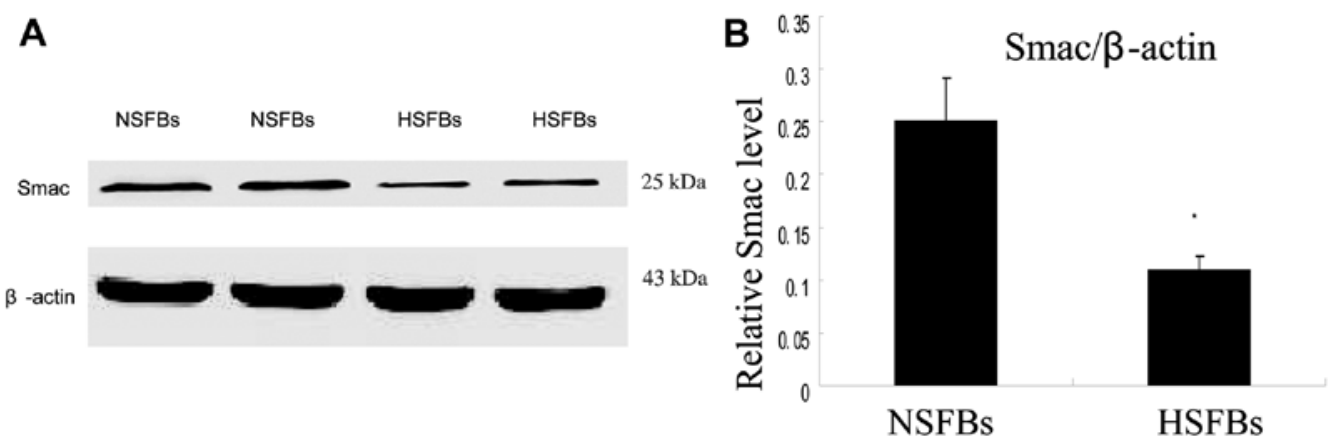

Figure 2. Cellular Smac protein expression level in hypertrophic scar fibroblasts (HSFBs) and normal skin fibroblasts (NSFBs). The expression of Smac protein was detected by western blot analysis in the HSFBs and NSFBs. (A) Example of typical expression of Smac and $\beta$-actin in cultured HSFBs and NSFBs. Ratios of the densities of Smac to $\beta$-actin measured using ImageJ software. (B) Results obtained show significantly lower Smac protein levels in the HSFBs compared with the NSFBs $\left({ }^{*} \mathrm{P}<0.01\right)$.

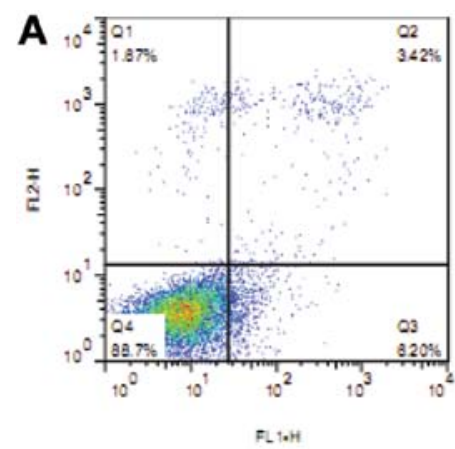

D

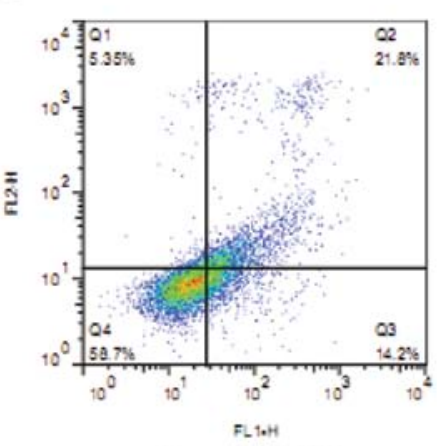

Control siRNA

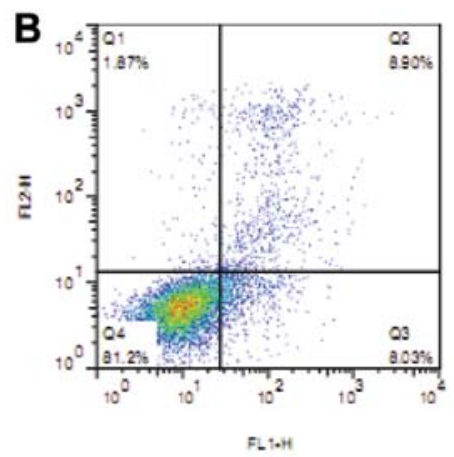

E

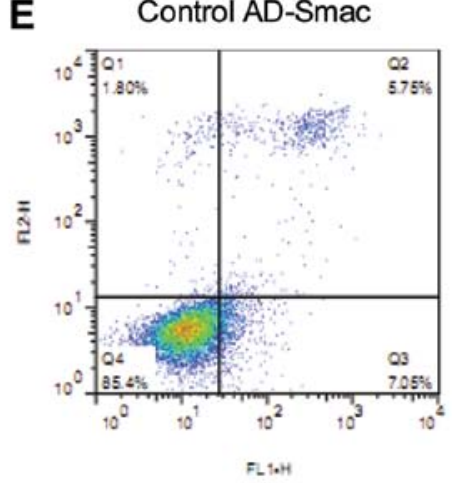

Smac siRNA

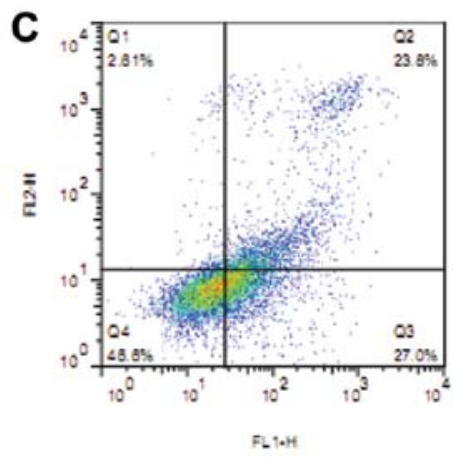

AD-Smac

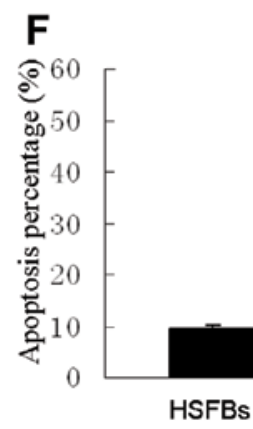

Figure 3. Analysis of cell apoptosis. (A-E) Flow cytometric analysis of the number of apoptotic cells in the hypertrophic scar fibroblast (HSFB) group, control AD-Smac group (adenovirus only), AD-Smac group (adenovirus overexpressing Smac/DIABLO), control siRNA group and siRNA group. (F) The number of apoptotic cells was significantly increased in the AD-Smac group compared with the control AD-Smac group ( $\left.{ }^{* *} \mathrm{P}<0.01\right)$. No significant changes were observed between the HSFB group and the control AD-Smac group, whereas the apoptotic rate in the siRNA group was significantly lower compared with that in the control siRNA group $\left({ }^{*} \mathrm{P}<0.01\right)$.

expression in HSFBs. The apoptotic rate of the HSFB group, as measured by flow cytometry, was the lowest among the
5 groups (Fig. 3). The apoptotic rates of the control AD-Smac group and the control siRNA group were higher than the 


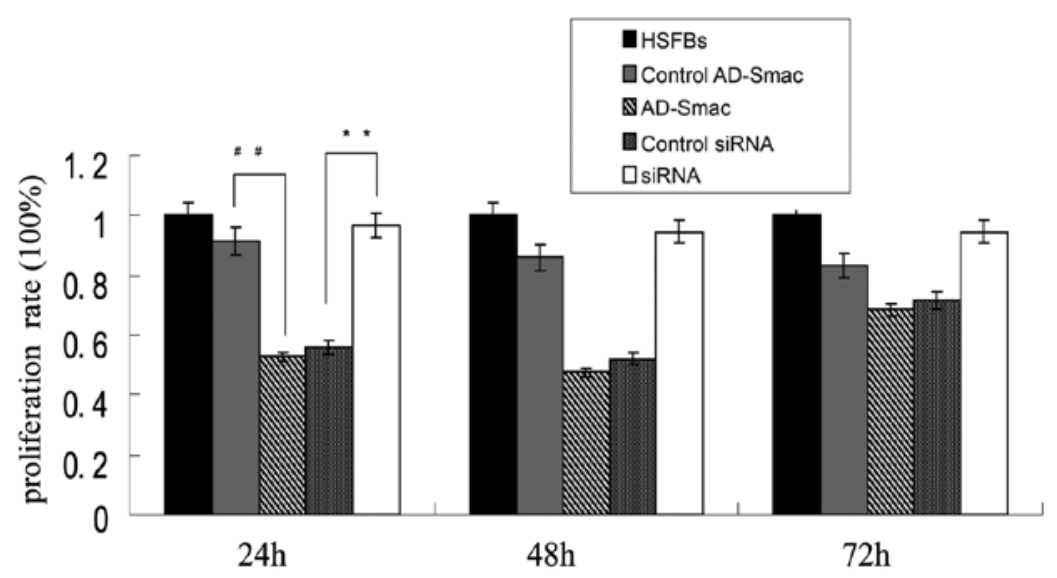

Figure 4. Measurement of the cell proliferation rate using a cell counting kit-8 (CCK-8) assay after 24,48 and $72 \mathrm{~h}$. The relative proliferation rates of the control AD-Smac group (transfected only with the adenovirus), AD-Smac group (overexpressing Smac), control siRNA group and siRNA group (suppression of Smac expression) are all lower than the rate in the hypertrophic scar fibroblast (HSFB group). These results suggest that Smac overexpression significantly inhibited HSFB proliferation, whereas Smac siRNA induced the opposite effect and significantly enhanced HSFB proliferation at 24 and $48 \mathrm{~h}$. However, the differences in proliferation rate were reduced with time $(72 \mathrm{~h})$.

HSFB group. Moreover, the apoptotic rate of the AD-Smac group, which overexpressed Smac, was significantly higher than that of the control AD-Smac group, transfected with the adenovirus only $(\mathrm{P}<0.01)$. The apoptotic rate of the control siRNA group was significantly higher than that of the siRNA group $(\mathrm{P}<0.01)$. These results suggest the possible role of Smac in the induction of apoptosis in HSFBs.

Smac overexpression significantly inhibits HSFB proliferation. The proliferation rate of the HSFBs was regarded as $100 \%$ and the proliferation of all the other groups was lower than that ofs the HSFBs (Fig. 4). A significant suppressive effect on cell proliferation was observed following the transfection of the HSFBs with AD-Smac, as shown by CCK-8 assay. A marked decrease in proliferation was observed in the cells overexpressing Smac (transfected with AD-Smac) (52.86\%) compared with the control AD-Smac-transfected cells $(91.66 \%)$ at $24 \mathrm{~h}$ $(\mathrm{P}<0.01)$. The proliferation rate of the siRNA group was significantly increased $(96.64 \%)$ compared with that of the control siRNA group $(56.03 \%)$ at $24 \mathrm{~h}(\mathrm{P}<0.01)$. The differences in the 2 proliferation rates at $24 \mathrm{~h}$ were greater than at 48 and $72 \mathrm{~h}$. Therefore, according to these results, it can be concluded that Smac overexpression significantly inhibited HSFB proliferation, while the silencing of Smac by siRNA induced HSFB proliferation.

Smac overexpression downregulates the mRNA levels of type I and III pro-collagen. The excessive synthesis of type I and III pro-collagen is an important characteristic of HS. We therefore assessed the mRNA expression levels of type I and III procollagen in the HSFBs following treatment with AD-Smac or control AD-Smac and siRNA, or control siRNA. The results revealed no significant differences in the mRNA levels of type I and III pro-collagen among the HSFB group, the control AD-Smac group and the control siRNA group $(\mathrm{P}>0.05)$. The levels of type I and III pro-collagen were significantly decreased in the cells overexpressing Smac (AD-Smac group) compared with the control AD-Smac group and the HSFB group. The levels were upregulated in the siRNA group, where
Smac expression was suppressed, compared with the control siRNA group and the HSFB group. These results demonstrate that Smac overexpression downregulates the mRNA levels of type I and III pro-collagen in the HSFBs. It was also shown that Smac preferentially affects the mRNA levels of type I pro-collagen compared with the mRNA levels of type III procollagen (Fig. 5).

Smac overexpression enhances the activity of caspase-3 and -9 in HSFBs. Caspase-3 and -9 are 2 important members of the caspase family and caspase- 3 activation is considered as the executing event of apoptosis. Caspase- 3 and -9 activity, as dectected by spectrofluorimetry, was activated in the HSFBs following transfection with AD-Smac (Fig. 6). The activity of caspase-3 and -9 was 3-fold greater in the HSFBs overexpressing Smac (AD-Smac group) than in the control HSFBs (HSFB group) or the control AD-Smac-transfected group. The activityof caspase-3 and -9 was markedly reduced (by 50\%) in the siRNA group, where Smac expression was suppressed, compared with the HSFB group and control siRNA group.

\section{Discussion}

When severe trauma or deep burns occur on the skin, wound healing is crucial in order to restore the cutaneous barrier. Blood platelet cells, inflammatory cells, endothelial cells, fibroblasts and keratinocytes are recruited to prevent bleeding and promote wound healing. These cells form new blood vessels and produce ECM to create a new layer covering the surface of the wound. The majority of shallow wounds heal within a few days. However, in some individuals, the dermal fibroblasts proliferate excessively and secrete an overabundant ECM, resulting in HS. HS are clinically described as raised, pruritic and erythematous fibrous lesions limited within the boundary of the original wound. HS lesions are often bulky and inelastic scars which can severely restrict the mobility of joints and extremities, immobilize structures, constrict orifices and drastically compromise cosmetic appearance (2). Increased HSFB proliferation and decreased HSFB apoptosis 


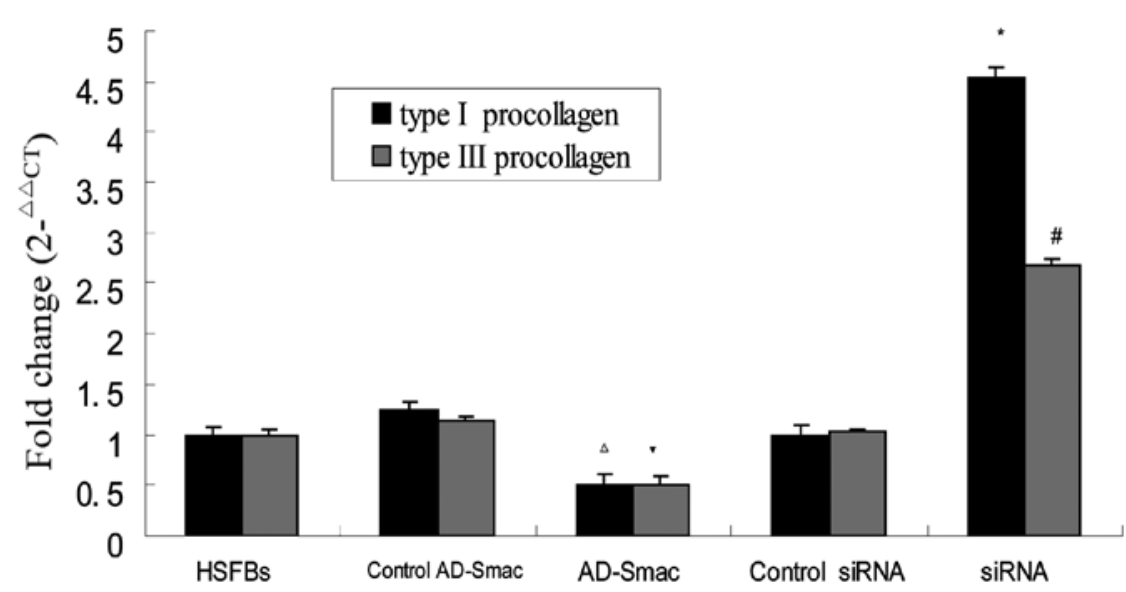

Figure 5. Effects of Smac overexpression on mRNA levels of type I and III pro-collagen. The overexpression of Smac downregulated the mRNA levels of type I and III pro-collagen, whereas Smac siRNA significantly upregulated the mRNA levels of type I and III pro-collagen. Remarkably, the increase in type I procollagen was higher than the increase in type III pro-collagen. ${ }^{\wedge} \mathrm{P}<0.01$ vs. control AD-Smac; ${ }^{\top} \mathrm{P}<0.01$ vs. control AD-Smac; ${ }^{*} \mathrm{P}<0.01$ vs. control siRNA; ${ }^{*} \mathrm{P}<0.01$ vs. control siRNA.

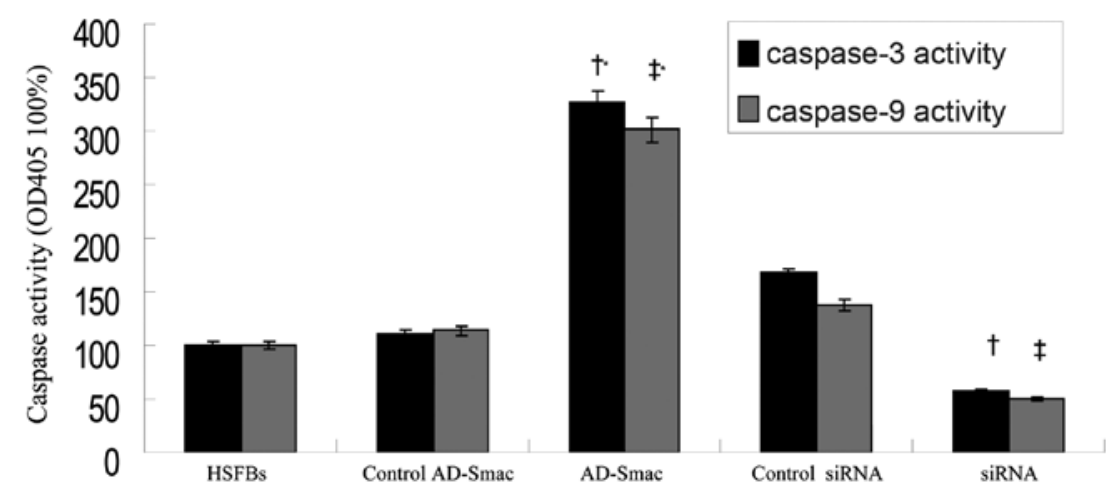

Figure 6. Effects of Smac overexpression and suppression of Smac expression on caspase-3 and -9 activity. Caspase activity was measured using Ac-DEVD-AFC as a caspase-3 substrate or Ac-LEHD-AFC as a caspase-9 substrate in hypertrophic scar fibroblasts (HSFBs) transfected with the adenovirus carrying Smac or the control and in the HSFBs transfected with siRNA against Smac or control siRNA; the caspace activity of the control was $100 \%$. Smac overexpression significantly increased the activity of caspase- 3 and -9 (AD-Smac group). Significant differences in caspase- 3 and -9 activity were not observed among the HSFB group, control AD-Smac group and control siRNA group. ${ }^{\dagger *} \mathrm{P}<0.01$ vs. control AD-Smac; ${ }^{* *} \mathrm{P}<0.01$ vs. control AD-Smac ; ${ }^{\dagger} \mathrm{P}<0.01$ vs. control siRNA; ${ }^{\dagger} \mathrm{P}<0.01$ vs. control siRNA.

have been suggested to be the main factors in the development of HS, whereas the upstream regulators of apoptosis in the wound environment remain unclear (19-21). Among the numerous key factors of wound healing (22), of all proteins involved in the apoptotic processes $(23,24)$, the differences in their expression and distribution in the wound healing process (25), a number of targets have been suggested to improve healing without keloids or HS (22-24). However, little is known about the effects of Smac/DIABLO on skin wound healing processes; it is currently accepted that the expression levels of Smac/DIABLO are downregulated in several excessive proliferative diseases or tumors compared with normal tissues (13-18).

In this study, we investigated the expression level of Smac/ DIABLO in HSFBs and normal fibroblasts. We observed a significant downregulation of Smac/DIABLO expression in HS compared to normal skin tissues using both immunohistochemical labeling and western blot analyses. Thus, our results on HS processes, similar to other proliferative diseases or tumors (13-18), suggest the important role of Smac/DIABLO in the process of HS, mainly through the regulation of HSFB apoptosis and proliferation.

In order to confirm this hypothesis, we induced the overexpression of Smac in HSFBs. The overexpression of Smac significantly increased the apoptotic rate of the HSFBs and thus inhibited their proliferation. Remarkably, the transfection of these cells with Smac siRNA, suppressing Smac expression, had the opposite effect, inducing a significant reduction in apoptosis and a restoration of the HSFB proliferation rate. Consistent with the data from previous studies on various proliferative diseases $(26,27)$, our results clearly demonstrate that Smac/DIABLO plays an important role in the regulation of apoptosis and proliferation of cells. However, the exact mechanism behind the Smac regulation of proliferation of HSFBs remains unknown.

The overexpression Smac/DIABLO by transfection of the cells with adenovirus carrying Smac increased caspase-3 and -9 activity. Restoration of the caspase rate (to low levels) with Smac siRNA suggests the major role played by Smac/ DIABLO in the complex regulation of HSFB proliferation 
during wound healing. According to previous studies (28-30), apoptosis caused by Smac in the HSFBs mainly depends on the caspase pathways. Following the induced overexpression of Smac in HSFBs and its inhibition by Smac siRNA, our results strongly suggest that the intrinsic pathway of apoptosis is one of the main regulators of fibroblast function, i.e., colonization and proliferation, during skin wound healing.

However, HS is also characterized by a decrease in collagenase content, an increased collagen synthesis mainly by fibroblasts, leading to an abnormal collagen deposition and atypical ECM remodeling in the scar tissue (31). The ECM in the skin is mainly characterized by the presence of type I and III pro-collagen, whose expression is significantly increased in HS. Remarkably, the expression level of type III pro-collagen is lower than that of type I pro-collagen in keloid scars. In a previous study, Oliveira et al (32) found that type III pro-collagen levels were significantly increased in HS compared to non-HS, whereas no difference in type I pro-collagen levels were observed in the same samples. Thus, the excessive secretion of type I pro-collagen also appears to play an important role in HS, and may be involved in the severity of HS. Thus, it would be of importance to find exogenous factors affecting collagen synthesis for the treatment of scarring. For example, many treatments with growth factors $(33-35)$, steroids $(36,37)$ or natural products $(38,39)$ may not only regulate the expression of collagens, but also collagen bundle organization. In this study, we investigated whether, in addition to its effects on HSFB proliferation and apoptosis, the expression of Smac can influence collagen type I and III expression. Our results demonstrated that Smac overexpression inhibited the mRNA expression of type I and III pro-collagen in the HSFBs. Indeed, the effects of Smac overexpression on the mRNA levels of type I pro-collagen were more significant than those on the mRNA levels of type III pro-collagen. Thus, the overproduction of collagen and atypical ECM remodeling in the scar tissue, with the modification of the ratio collagen I/III, lead to fibrosis of the scar. These results suggest for the first time a possible link (direct or indirect) between Smac/DIABLO and the levels of type I and III collagen in fibroblasts, which may be involved in the formation of HS.

In conclusion, to our knowledge, in this study, we demonstrate for the first time that in HS tissue, Smac/DIABLO is downregulated compared to NSFBs. This results not only in the promotion of fibroblast proliferation or in the reduction of their sensitivity to apoptotic signals, but also in the increase in type I and III pro-collagen expression. As all these events were inhibited by Smac overexpression, this suggests that Smac/ DIABLO may be a novel therapeutic target that may be used to prevent and control HS formation. Finally, the regulation of Smac/DIABLO may provide a potential approach for the regulation and improvement of skin wound healing.

\section{Acknowledgements}

We are particularly grateful to Tao Wang and Lilong Zhang for their technical assistance (College of Preventive Medicine, Third Military Medical University, Chongqing 400038 , China). This study was supported by the National Natural Science Foundation of China (project no. 81272102).

\section{References}

1. O'Leary R, Wood EJ and Guillou PJ: Pathological scarring: strategic interventions. Eur J Surg 168: 523-534, 2002.

2. van der Veer WM, Bloemen MC, Ulrich MM, et al: Potential cellular and molecular causes of hypertrophic scar formation. Burns 35: 15-29, 2009.

3. De Felice B, Garbi C, Santoriello M, et al: Differential apoptosis markers in human keloids and hypertrophic scars fibroblasts. Mol Cell Biochem 327: 191-201, 2009.

4. Greenhalgh DG: The role of apoptosis in wound healing. Int J Biochem Cell Biol 30: 1019-1030, 1998.

5. Desmouliere A, Badid C, Bochaton-Piallat ML and Gabbiani G: Apoptosis during wound healing, fibrocontractive diseases and vascular wall injury. Int J Biochem Cell Biol 29: 19-30, 1997.

6. Linge C, Richardson J, Vigor C, et al: Hypertrophic scar cells fail to undergo a form of apoptosis specific to contractile collagen-the role of tissue transglutaminase. J Invest Dermatol 125: 72-82, 2005.

7. Akasaka Y, Fujita K, Ishikawa Y, et al: Detection of apoptosis in keloids and a comparative study on apoptosis between keloids, hypertrophic scars, normal healed flat scars, and dermatofibroma. Wound Repair Regen 9: 501-506, 2001.

8. Tanaka A, Hatoko M, Tada $\mathrm{H}$, et al: Expression of p53 family in scars. J Dermatol Sci 34: 17-24, 2004.

9. Aarabi S, Bhatt KA, Shi Y, et al: Mechanical load initiates hypertrophic scar formation through decreased cellular apoptosis. FASEB J 21: 3250-3261, 2007.

10. Du C, Fang M, Li Yucheng, et al: Smac, a mitochondrial protein that promotes cytochrome $c$-dependent caspase activation by eliminating IAP inhibition. Cell 102: 33-42, 2000.

11. Verhagen AM, Ekert PG, Pakusch M, et al: Identification of DIABLO, a mammalian protein that promotes apoptosis by binding to and antagonizing IAP proteins. Cell 102: 43-53, 2000.

12. Mao HL, Liu PS, Zheng JF, et al: Transfection of Smac/DIABLO sensitizes drug-resistant tumor cells to TRAIL or paclitaxelinduced apoptosis in vitro. Pharmacol Res 56: 483-492, 2007.

13. Kempkensteffen C, Hinz S, Christoph F, et al: Expression levels of the mitochondrial IAP antagonists Smac/DIABLO and Omi/ HtrA2 in clear-cell renal cell carcinomas and their prognostic value. J Cancer Res Clin Oncol 134: 543-550, 2008.

14. Mizutani Y, Nakanishi H, Yamamoto K, et al: Downregulation of Smac/DIABLO expression in renal cell carcinoma and its prognostic significance. J Clin Oncol 23: 448-454, 2005.

15. Sekimura A, Konishi A, Mizuno K, et al: Expression of Smac/ DIABLO is a novel prognostic marker in lung cancer. Oncol Rep 11: 797-802, 2004.

16. Kempkensteffen C, Jager T, Bub J, et al: The equilibrium of XIAP and Smac/DIABLO expression is gradually deranged during the development and progression of testicular germ cell tumours. Int J Androl 30: 476-483, 2007.

17. Bao ST, Gui SQ and Lin MS: Relationship between expression of Smac and Survivin and apoptosis of primary hepatocellular carcinoma. Hepatobiliary Pancreat Dis Int 5: 580-583, 2006.

18. Martinez-Ruiz G, Maldonado V, Ceballos-Cancino G, et al: Role of Smac/DIABLO in cancer progression. J Exp Clin Cancer Res 27: 48, 2008

19. Sun Z, Li s, Cao C, et al: shRNA targeting SFRP2 promotes the apoptosis of hypertrophic scar fibroblast. Mol Cell Biochem 352: 25-33, 2011.

20. Guo L, Chen L, Bi S, et al: PTEN inhibits proliferation and functions of hypertrophic scar fibroblasts. Mol Cell Biochem 361: $161-168,2012$.

21. Cao C, Li S, Dai X, et al: Genistein inhibits proliferation and functions of hypertrophic scar fibroblasts. Burns 35: 89-97, 2009.

22. Mahdavian Delavary B, van der Veer WM, van Egmond M, et al: Macrophages in skin injury and repair. Immunobiology 216: 753-762, 2011.

23. Honardoust D, Ding J, Varkey M, et al: Deep dermal fibroblasts refractory to migration and decorin-induced apoptosis contribute to hypertrophic scarring. J Burn Care Res 33: 668-677, 2012.

24. Akasaka Y, Ono I, Kamiya T, et al: The mechanisms underlying fibroblast apoptosis regulated by growth factors during wound healing. J Pathol 221: 285-299, 2010.

25. Jiang L, Zhang E, Yang Y, et al: Effectiveness of apoptotic factors expressed on the wounds of patients with stage III pressure ulcers. J Wound Ostomy Continence Nurs 39: 391-396, 2012.

26. Jia L, Patwari Y, Kelsey SM, et al: Role of Smac in human leukaemic cell apoptosis and proliferation. Oncogene 22: 1589-1599, 2003. 
27. Chen DJ and Huerta S: Smac mimetics as new cancer therapeutics. Anticancer Drugs 20: 646-658, 2009.

28. Chai J, Du C, Wu JW, et al: Structural and biochemical basis of apoptotic activation by Smac/DIABLO. Nature 406: 855-862, 2000.

29. Wu G, Chai J, Suber TL, et al: Structural basis of IAP recognition by Smac/DIABLO. Nature 408: 1008-1012, 2000.

30. Gao Z, Tian Y, Wang J, et al: A dimeric Smac/diablo peptide directly relieves caspase-3 inhibition by XIAP. Dynamic and cooperative regulation of XIAP by Smac/Diablo. J Biol Chem 282: 30718-30727, 2007.

31. Clark JA, Cheng JC and Leung KS: Mechanical properties of normal skin and hypertrophic scar. Burns 22: 443-446, 1996.

32. Oliveira GV, Hawkins HK, Chinkes D, et al: Hypertrophic vs. non hypertrophic scars compared by immunohistochemistry and laser confocal microscopy: type I and III collagens. Int Wound J 6: 445-452, 2009.

33. Akita S, Akino K, Tanaka K, et al: A basic fibroblast growth factor improves lower extremity wound healing with a porcinederived skin substitute. J Trauma 64: 809-815, 2008.

34. Cho JW, Kang MC and Lee KS: TGF- $\beta 1$-treated ADSCs-CM promotes expression of type I collagen and MMP-1, migration of human skin fibroblasts, and wound healing in vitro and in vivo. Int J Mol Med 26: 901-906, 2010.
35. Yan G, Sun H, Wang F, et al: Topical application of hPDGF-Amodified porcine BMSC and keratinocytes loaded on acellular HAM promotes the healing of combined radiation-wound skin injury in minipigs. Int J Radiat Biol 87: 591-600, 2011.

36. Weshahy $\mathrm{AH}$ and Abdel Hay R: Intralesional cryosurgery and intralesional steroid injection: a good combination therapy for treatment of keloids and hypertrophic scars. Dermatol Ther 25: 273-276, 2012

37. Hayashi T, Furukawa H, Oyama A, et al: A new uniform protocol of combined corticosteroid injections and ointment application reduces recurrence rates after surgical keloid/hypertrophic scar excision. Dermatol Surg 38: 893-897, 2012.

38. Wu JG, Ma L, Zhang SY, et al: Essential oil from rhizomes of Ligusticum chuanxiong induces apoptosis in hypertrophic scar fibroblasts. Pharm Biol 49: 86-93, 2010.

39. Morin C, Roumegous A, Carpentier G, et al: Modulation of inflammation by Cicaderma ointment accelerates skin wound healing. J Pharmacol Exp Ther 343: 115-124, 2012. 